\title{
The Characteristic Parameter Estimation of Low Temperature Target Weak Signal Based on VanderPol-Duffing System
}

\author{
Yanchun $\mathrm{Xu}^{1,2}$, Danfeng Zhao ${ }^{1}$ \\ College of Information and Communication Engineering \\ 1.Harbin Engineering University,Harbin,China, 150080 \\ 2. Heilongjiang University, Harbin, China, 150080 \\ xyc7309@163.com
}

\author{
Mi Lu \\ School of Electrical and Computing Engineering \\ Texas A\&M University,College Station, USA,77840 \\ mlu@ece.tamu.edu
}

\begin{abstract}
The weak signal, which is usually submerged in strong noise, is very difficult to detecte for its amplitude and frequency. The dynamic properties of VanderPol-Duffing are studied in this paper. Such system can go into the chaos under certain parameters. In chaotic state the disturbance of weak periodic signals can make the system dynamic behavior change dramatically. Our research results show that the system is from period doubling state to chaotic state when the amplitude of input signal is changed. And it has a remarkable impact influence on the system dynamic performance when the input frequency is varied. The unknown frequency can be detected through counting the numbers of turning point in phase diagram. The simulation results verified that the presented method is feasible and there are a lot of theory values in the research.
\end{abstract}

Keywords-VanderPol-Duffing equation; the weak signal detection; amplitude extracting; frequency estimation

\section{INTRODUCTION}

The technology of weak signal detection is applied for the field of optics, electronics, biology and so on ${ }^{[1-3]}$. The weak signal detection of low temperature target is a new research focus now. The absolute value of energy is smaller and the signal is weaker in the low temperature signal detection. Meanwhile temperature noise, thermal noise, background noise and so on affected the detection value in the actual measurement. The detected signal is usually submerged in complex noise. So far we can't detect the useful signal using the traditional device. So a new algorithm of weak signal detection should be researched and a new device should be developed.

There are a lot of traditional methods in the field of weak signal detection, such as narrow-band filtering, dual eliminating, lock receiver and so on. Although these methods can be used to detect weak signal, there are still vital shortages. For example, the center frequency is unsteady in normal narrow-band filter so that the high frequency noise can't be filtered. Also, the weak sinusoidal signal can be detected in dual eliminating method but the sinusoidal waveform can't be reproduced. Furthermore, the integration time of correlation detection method can't be infinitely long so there is some noise in the system. Recently chaotic theory is applied to weak signal detection and considerable progress has been achieved with the research improvement in chaotic theory ${ }^{[4-6]}$. Guanyu Wang used Duffing chaotic oscillator to detect weak sinusoidal signal in the presence of the strong noise, and obtained high-precision measurement results. Here, thevalidity of using Duffing system detection was evidenced. Yue $\mathrm{Li}$ used the modified Duffing equation to implement simulation of $\mathrm{nV}$ level signal detection, and the Melnikov method was used for the threshold value of chaotic oscillator, though the precision of the threshold value was limited. A method of weak signal detection based on Duffing oscillator is studied by Jianxiong Wang. It was introduced to use Melnikov function in determining the threshold of detection system. Xiuqiao Xiang and Baochang Shi presented a method using information fusion for weak signal detection and the method is based on the Duffing chaotic oscillator. Most research reports, however, were limited on the Duffing system for the weak signal detection instead of other chaotic systems due to its simpler structure and the convenience of study ${ }^{[7,8]}$. Nevertheless a 78 chaotic oscillator is needed to detect the unknown frequency of weak signal which is a lot of difficulties, shortage and exist in conducting weak signal detection.

In this paper VanderPol-Duffing system is used to detect weak signals. The parameter is adjusted in order to control the critical state of VanderPol-Duffing. Then the detected signal is put into the VanderPol-Duffing system. The amplitude of unknown weak signal can be detected through the change of phase diagram. At the same time the unknown frequency can be detected as well, via the observation of the number of inflection point. This method is simpler and easier to realize compared to the Duffing system.

\section{WEAK SIGNAL DETECTION BASED VANDERPOL-DUFFING SYSTEM}

A. The chaotic charicteristics of vanderpol-duffing system

The VanderPol-Duffing equation is

$$
\begin{gathered}
\ddot{x}-\delta\left(1-x^{2}\right) \dot{x}+\alpha x+\beta x^{3}=A \cos \omega t \\
\text { That is }\left\{\begin{array}{c}
\dot{x}=y \\
\dot{y}=\delta\left(1-x^{2}\right) \dot{x}+\alpha x+\beta x^{3}
\end{array}\right.
\end{gathered}
$$


We proposed to set the parameters $\delta=0.2, \alpha=0, \beta=1$, $A=17, \omega=4$ and under such condition the system enter the chaotic state. An extra cube term is involved in VanderPol-Duffing system compared to VanderPol system which made the chaotic properties more complex. It shows that even though the value of $\beta$ is small, the nonlinear term $\beta x^{3}$ can affect the chaotic state dramatically.

When the parameter $\beta$ is zero, the system is going into the chaotic state. However the chaotic state is disappeared as we considered the nonlinear term $\beta x^{3}$. The results show that the term $\beta x^{3}$ should not be made linear if one needs the chaotic state in some condition. The reason is that it can change the property of the system.

Fig. 1 shows that the Vander Pol-Duffing system is affected when the parameters are changed. It is the simulation results given the parameters $\alpha=1, \beta=0.1, A=5, \omega=4$.
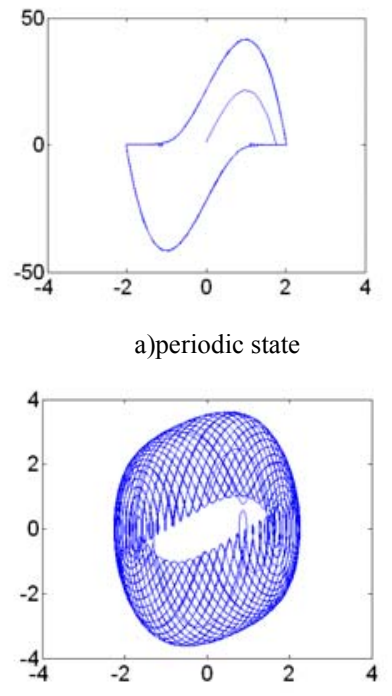

b)period bifurcation

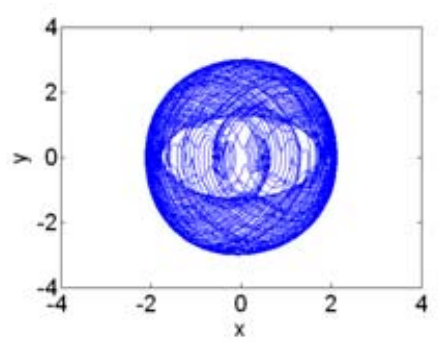

c) chaotic state

Figure. 1 the phase diagram of VanderPol-Duffing system

The VanderPol-Duffing system is a periodic state when the parameter is set to $\delta=30$ and the system is periodic bifurcation when the parameter is set to $\delta=1$.
The bifurcation will continue the parameter $\delta$ is reduced and the system will get into chaotic state finally. It shows that the VanderPol-Duffing system is dependent on the initial value and state. The VanderPol-Duffing system is sensitive to the weak signal, hence can be utilized for the weak signal detection.

\section{B. The detection of signal amplitude}

The chaotic system is very sensitive to the parameter change of the system. The system behavior can be changed obviously while the unknown signal amplitude is changed microscopically. If the amplitude and frequency of standard signal $A_{0} \cos \omega_{0} t$ are known and put into the chaotic system, the parameter $\delta$ is controlled in order to make the system become critical chaotic state. The unknown signal is then put into the critical chaotic system. If a noise is put into the system has an immunity and the state has no change. But if the unknown signal $S=A \cos \left(\omega_{0} t\right)$ is put into the VanderPol-Duffing chaotic system as the periodic driving motivation, the system state is changed dramatically.

That is,

$$
\ddot{x}-\delta\left(1-x^{2}\right) \dot{x}+\alpha x+\beta x^{3}=A_{0} \cos \omega_{0} t+A \cos \omega t
$$

Where $A_{0} \cos \omega_{0} t$ is the periodic driving force and $A \cos \omega t$ is unknown weak signal submerged into the noise. If the frequency of unknown signal and the frequency of periodic driving force are the same, the system can be written as follows.

$$
\ddot{x}-\delta\left(1-x^{2}\right) \dot{x}+\alpha x+\beta x^{3}=\left(A_{0}+A\right) \cos \omega_{0} t
$$

When parameters $\alpha, \beta$ and $\delta$ are fixed, $A_{0}$ is regulated until the total amplitude $\left(A_{0}+A\right)$ makes the system become the critical state of chaotic system. Then

$$
\Delta A=f_{d}-A_{0} .
$$

Here, parameter $\Delta A$ is the detected amplitude.

Fig. 2 is the chaotic phase diagram when the parameter is $A_{0}=0.65$.

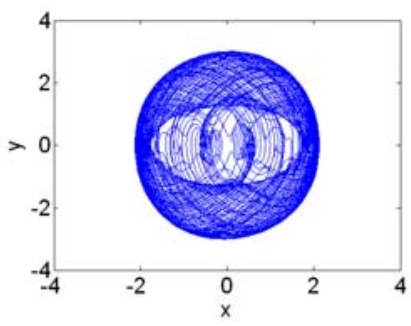

Figure. 2 Chaotic state

Fig. 3 is the large periodic state when the parameter is $A_{0}=0.677$. 


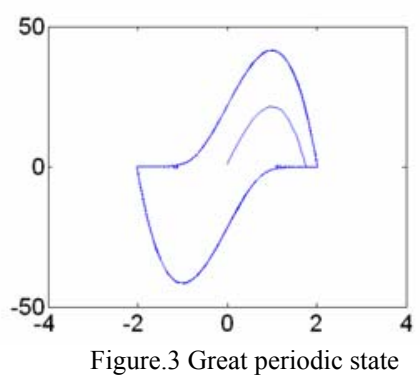

In order to simplify the system the unknown weak signal is used as driving force. That is,

$$
\ddot{x}-\delta\left(1-x^{2}\right) \dot{x}+\alpha x+\beta x^{3}=m[A \cos t+n(t)] .
$$

Then regulate the parameter $m$ in order to increase the amplitude of unknown weak signal until the phase diagram is changed and find the critical value of the system $f_{d}$. The parameter $m_{d}$ is written down and the equation is $m_{d} A=f_{d}$. Through this equation the amplitude of weak signal $A$ is obtained.

\section{The frequency detection based on vanderpol- duffing system}

The model of VanderPol-Duffing system is $\ddot{x}-$ $0.01\left(1-x^{2}\right) \dot{x}+1 \cdot x+0.1 x^{3}=A \cos \omega t$. This system is sensitive to the unknown frequency of weak signal so it can be used to detect the unknown frequency. When the parameters are $\delta=0.01, \alpha=1$ and $\beta=0.1$, the simulation results show that given the input signal cost, the system is in the times periodic state. If the frequency is changed $2 t$, the phase diagram of chaotic system is of secondary distortions. Similar simulations occur to $3 t, 4 t, 5 t \cdots \cdots$, the phase diagram will become the same times distortions. The number of distortions will be the frequency of the unknown signal. The frequency can be obtained by counting the numbers of inflection point.

However the method is invalid if the frequency is not

\section{SYSTEM SIMULATIONS}

When the frequency of weak signal is known, the amplitude of weak signal can be detected. But if the frequency is unkown, we need to detect the frequency first and then to detect the amplitude using VanderPolDuffing system.

\section{A. The simulations of frequency detection based on vanderpol-duffing system}

In the simulation, the unknown signal is used as the driving force and the amplitude of weak signal is 0.06 . The frequency is $1 \mathrm{rad} / \mathrm{s}, 3 \mathrm{rad} / \mathrm{s}, 4 \mathrm{rad} / \mathrm{s}$ and $6 \mathrm{rad} / \mathrm{s}$ respectively. The simulation results are shown in Fig.4. integer. So the Duffing system can be used to detect unknown frequency. Many geometric chaotic oscillators can be used to detect any frequency of weak signals. In fact, most of the frequencies are known and even if it's unknown, most of them are the integer frequency. So the presented method is very valuable to detect weak signal frequency in engineering field.

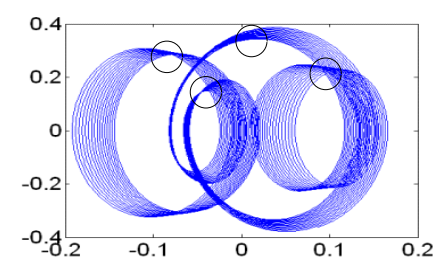

c) $\omega=4 \mathrm{rad} / \mathrm{s}$

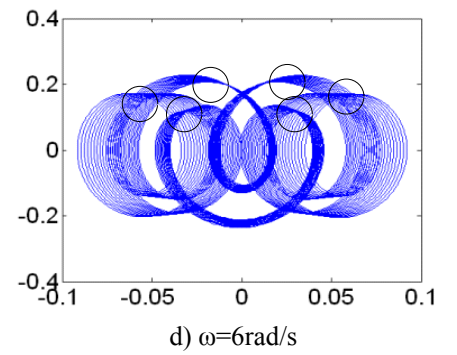

Figure. 4 the phase diagram of the frequency detection based on VanderPol-Duffing system

\section{B. The simulations of amplitude detection based on vanderpol-duffing system}

The phase diagram is shown in Fig.5 when the amplitude of weak signal is varied finely and the parameter $\delta$ is 0.2 . At the Same time the noise is zeromean. It is shown in Fig. 5 it shows that the dynamic behavior is changed a lot when the amplitude of weak signal varies from 0.65 to 0.651 .

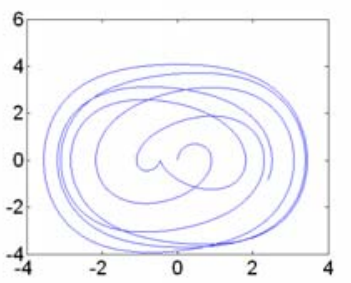

a) $\omega=1 \mathrm{rad} / \mathrm{s}$

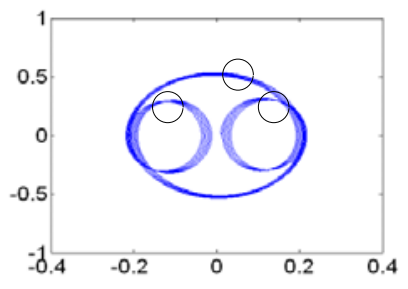

b) $\omega=3 \mathrm{rad} / \mathrm{s}$ 


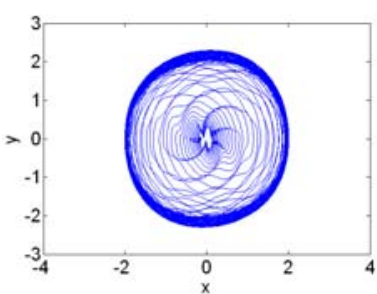

a)the amplitude is 0.65

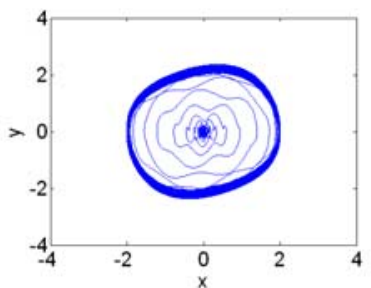

b)the amplitude is 0.651

Figure.5 the influence to VanderPol-Duffing system when amplitude changes

It demonstrates that the weak signal can change the dynamic state of the chaotic system when the system is under critical state. The amplifying function of weak signal can detect the amplitude of the unknown weak signal.

Obviously, the two phase diagrams are different in Fig.5. The former is more chaotic and the reason is the periodic bifurcation is larger than one of the later.

The phase diagram of VanderPol-Duffing system is shown in Fig. 6 parameter $\delta$ being 0.02 and the noise is very strong. It shows that when the parameter $\delta$ is different, the dynamic behaviors of the systems are not the same.
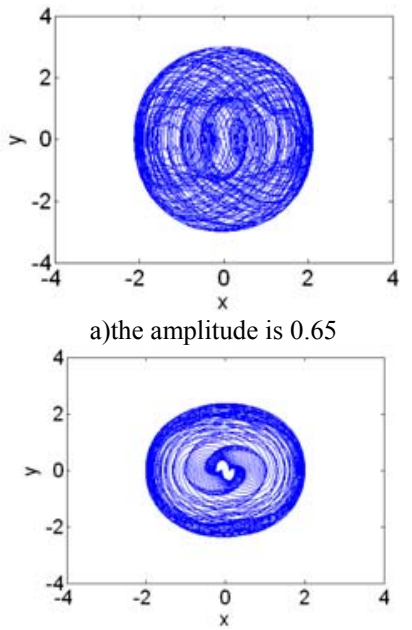

b) the amplitude is 0.68

Figure. 6 the influence toVanderPol-Duffing system of amplitude change when $\delta=0.02$

In the simulation the amplitude $A$ of weak signal is supposed to be 0.05 and the parameters are $\omega=2, \delta=$ $0.02, \alpha=1$ and $\beta=0.1$. Then the parameter $f_{d}$ is equal to 0.677 . When the parameter $m$ is 13.5 , the system goes from chaotic state to large periodic state. So the equation is $13.5 A=0.677$. The value of $A$ is gotten and it is 0.051 . The error is about $2 \%$.

A lot of simulation are done and to compare the presented method with one of the Duffing systems. The results are shown in Table 1.

Table 1. the comparison result in two detection methods

\begin{tabular}{ccccc}
\hline \multirow{2}{*}{$\begin{array}{l}\text { Signal- } \\
\text { noise ratio }\end{array}$} & \multicolumn{2}{c}{ VanderPol-Duffing system } & \multicolumn{2}{c}{ Duffing system } \\
\cline { 2 - 5 } & $\begin{array}{c}\text { Detected value } \\
(\mathrm{V})\end{array}$ & $\begin{array}{c}\text { error } \\
(\%)\end{array}$ & $\begin{array}{c}\text { Detected value } \\
(\mathrm{V})\end{array}$ & $\begin{array}{c}\text { error } \\
(\%)\end{array}$ \\
\hline-30 & 0.000501 & 0.2 & 0.000509 & 1.8 \\
\hline-40 & 0.000499 & -0.2 & 0.000487 & -2.6 \\
\hline-50 & 0.000502 & 0.4 & 0.000511 & 2.2 \\
\hline-60 & 0.000506 & 1.2 & 0.000482 & -3.6 \\
\hline-70 & 0.000489 & -2.2 & 0.000535 & 7.0 \\
\hline-77 & 0.000471 & -5.8 & 0.000421 & -15.8 \\
\hline-80 & 0.000468 & -6.4 & - & - \\
\hline
\end{tabular}

From Table 1 we can know that the presented method is feasible to detect the unknown frequency of the weak signal which is submerged in the strong noise. Meanwhile this method is superior to Duffing system and achieves a better accuracy.

\section{CONCLUSIONS}

In this paper the VanderPol-Duffing system is analized and is applied to the weak signal detection. The parameters are regulated and then the system turns to the critical state. At that moment the unknown weak signal is put into the chaotic system and the amplitude and frequency of weak signal detection are realized by examining a phase diagram. The simulation results show that the presented method can be efficiently used to detect the unknown weak signal and its accuracy is higher than that of the Duffing system. It has been verified that the 
presented method is effective and feasible.

\section{REFERENCES}

[1] Guanyu Wang, The application of chaotic oscillators to weak signal detection[C], IEEE Transaction on Industrial Electronics, 1999, 46(2): 440-444.

[2] R. S. Mackay and C. Tresser, Transition to topological chaos for circle maps [J], Physics D, 1986, 19: 206-237.

[3] C. Y. Song, Y. H. Lei and S. Q. Ding, Application of chaos to weak signal detection[J]. Editio 2004, 25: 21-23.

[4] C. U. Choe, K. Hohne, H. Benner, Chaos suppression in the parametrically driven Lorenz system [J], Physical Review.2005, 72(5): 362-366

[5] Guanyu Wang, Sailing He, A quantitative study on detection and estima tion of weak signals by using chaotic doffing oscillators [C], IEEE Transactions on Circuits and Systems-Fundamental Theory and Applications, 2003, 50(7): 945-953.

[6] Y. S. Wang and J. G. Yan, Capability of weak signal detection through chaos systems sensitive dependence on initial condition $[\mathrm{J}]$, Chinese Journal of Electron Devices, 2007, 30(5): 1650 1655.

[7] W. S. Yi, Y. W. Shi and C. Y. Nie, The chaotic oscillator estimate method for sin wave parameter in non-gaussian color noise environment[C], The Sixth International Conference on Electronic Measurement and Instrument,

Taiyuan, China. 2003, 4 (1):151 155.

[8] K. S. Helle and L. O. Chua, Signal amplification via chaos, Experimental Evidence[J], International Journal of Bifurcation and Chaos, 2006, 2(4):1008 1012. 